\title{
Repeat hepatectomy for recurrent colorectal cancer liver metastases after two-stage hepatectomy-limitations and opportunities
}

\author{
Hop S. Tran Cao, Jean-Nicolas Vauthey \\ Department of Surgical Oncology, The University of Texas MD Anderson Cancer Center, Houston, TX, USA \\ Correspondence to: Jean-Nicolas Vauthey, MD. Department of Surgical Oncology, The University of Texas MD Anderson Cancer Center, 1515 \\ Holcombe Boulevard, Unit 1484, Houston, TX 77030, USA. Email: jvauthey@mdanderson.org. \\ Comment on: Imai K, Benitez CC, Allard MA, et al. Impact of surgical treatment for recurrence after 2-stage hepatectomy for colorectal liver \\ metastases, on patient outcome. Ann Surg 2019;269:322-30.
}

Submitted May 31, 2019. Accepted for publication Jun 11, 2019.

doi: 10.21037/hbsn.2019.06.07

View this article at: http://dx.doi.org/10.21037/hbsn.2019.06.07

Whether resection of recurrent colorectal cancer liver metastases (CLMs) can be safely performed after prior hepatectomy, especially after two-stage hepatectomy (TSH), and to what extent this aggressive surgical approach may impact patient outcomes and prolong survival, are important issues to investigate. To this end, Imai and colleagues recently published the Hospital Paul Brousse experience with resection of recurrent CLMs after TSH (1). In this study, the authors analyzed 93 patients who underwent TSH, among whom 81 had "globally curative" surgery, when all known disease-including extrahepatic disease if present-was successfully resected. Of these 81 patients, $62(76.5 \%)$ experienced recurrence. For their entire study cohort, repeat liver surgery was ultimately performed in more than half of the patients, and survival for these patients was significantly greater than that for patients who did not undergo resection $(45.8 \%$ vs. $26.3 \%$, $\mathrm{P}=0.004)$. In fact, repeat hepatectomy for recurrence was the strongest independent predictor for improved survival. As the authors conclude, these results underscore the value of oncologic surveillance and the critical role of repeat hepatectomy. The present work expands on the concept of "third hepatectomy" previously reported by the same authors, focusing on those patients who have undergone the most aggressive surgical treatment of their prior CLMs in the form of TSH involving portal vein embolization and major hepatectomy (2). The demonstration of safety and efficacy of repeat hepatectomy in this setting provides critical information to support ongoing aggressive surgical strategies for this subset of patient.

It is unclear whether the current findings can be extrapolated to associating liver partition and portal vein ligation for staged hepatectomy (ALPPS), as suggested by the authors. In their recent publication comparing ALPPS to TSH (3), ALLPS resulted in a greater rate of resection by inducing more rapid and significant growth of the future liver remnant when compared to TSH, but it was associated with worse oncologic outcomes, with worse survival and recurrences that tended to be larger, more often found in the liver, and less amenable to repeat "salvage" hepatectomy. This more aggressive recurrence pattern, thought by some to be due to acceleration of residual tumor cell progression related to fast induction of hypertrophy in the liver (4), combined with its still nascent role in the treatment of CLMs, represent barriers to its widespread implementation.

Although the analysis of Imai et al. included certain aspects reflective of tumor biology, such as number and largest size of original CLMs, carcinoembryonic antigen level, and response to first-line therapy (or lack thereof), it did not offer details on the mutational profile of the tumors. In recent years, the critical influence of tumor genetic mutations on prognosis and oncologic outcomes for patients with colorectal cancer has come to light. In particular, mutations in the EGFR pathway, including those of the $R A S$ genes, have been shown to negatively impact survival and influence patterns of disease recurrence for patients with colorectal cancer $(5,6)$. Moreover, we have previously shown that $R A S$ mutations were associated with a greater 
rate of unresectable recurrence following hepatectomy for colorectal cancer liver metastases (7). With nearly 3 in 4 patients experiencing disease recurrence following TSH, understanding the impact of $R A S$ and other mutations on outcomes of repeat surgery in this setting may be critical to formulating effective treatment strategies.

To address this gap, our group recently published our own institutional data on resection of disease recurrence after TSH (8). In addition to assessing RAS mutation status, we also wanted to evaluate the potential role for surgical resection for any disease recurrence and not just those in the liver. In our series, 83 of 111 (75\%) patients who underwent TSH experienced disease recurrence, of whom nearly half (46\%) had $R A S$ mutant disease. Of the 83 patients with recurrence, 31 (37\%) were able to undergo resection, which was associated with improved survival. Conversely, $R A S$ mutation and first recurrence in multiple sites were independent predictors of worse survival. Fiveyear overall survival for patients who underwent surgery for $R A S$ wild-type was $86 \%$ compared to $38 \%$ for those with $R A S$ mutation. By comparison, survival for $R A S$ mutant patients treated non-operatively was only $8 \%$. These data highlight the following important points: (I) $R A S$ mutant status portends a worse prognosis and should be integrated in treatment planning; (II) even in this context, resection of disease recurrence may offer a survival benefit. These considerations are especially relevant for patients who undergo TSH, who typically have multiple small metastases and are prone to recurrences. In their treatment pathway, one must oftentimes decide whether to proceed with reresection or chemotherapy. $R A S$ mutational status might help in this decision, especially when the operation is deemed high-risk or complex, such as repeat hepatectomy after TSH.

We commend Imai et al. for their ongoing contributions to the care of patients with CLMs. Current approaches to this challenging clinical problem often require multiple hepatic resections, but must also consider tumor biology. As we seek to expand on this work, future studies on somatic mutations may contribute to improved selection and more effective treatment strategies, refining the role and timing of surgery and systemic therapy.

\section{Acknowledgments}

Funding: The MD Anderson Cancer Center support grant P30 CA016672.

\section{Footnote}

Conflicts of Interest: The authors have no conflicts of interest to declare.

Ethical Statement: The authors are accountable for all aspects of the work in ensuring that questions related to the accuracy or integrity of any part of the work are appropriately investigated and resolved.

\section{References}

1. Imai K, Benitez CC, Allard MA, et al. Impact of surgical treatment for recurrence after 2-stage hepatectomy for colorectal liver metastases, on patient outcome. Ann Surg 2019;269:322-30.

2. Adam R, Pascal G, Azoulay D, et al. Liver resection for colorectal metastases: the third hepatectomy. Ann Surg 2003;238:871-83; discussion 883-4.

3. Adam R, Imai K, Castro Benitez C, et al. Outcome after associating liver partition and portal vein ligation for staged hepatectomy and conventional two-stage hepatectomy for colorectal liver metastases. Br J Surg 2016;103:1521-9.

4. Oldhafer KJ, Donati M, Jenner RM, et al. ALPPS for patients with colorectal liver metastases: effective liver hypertrophy, but early tumor recurrence. World J Surg 2014;38:1504-9.

5. Conlin A, Smith G, Carey FA, et al. The prognostic significance of K-ras, p53, and APC mutations in colorectal carcinoma. Gut 2005;54:1283-6.

6. Vauthey JN, Zimmitti G, Kopetz SE, et al. RAS mutation status predicts survival and patterns of recurrence in patients undergoing hepatectomy for colorectal liver metastases. Ann Surg 2013;258:619-26; discussion 626-7.

7. Okuno M, Goumard C, Kopetz S, et al. RAS mutation is associated with unsalvageable recurrence following hepatectomy for colorectal liver metastases. Ann Surg Oncol 2018;25:2457-66.

8. Lillemoe HA, Kawaguchi Y, Passot G, et al. Surgical resection for recurrence after two-stage hepatectomy for colorectal liver metastases is feasible, is safe, and improves survival. J Gastrointest Surg 2019;23:84-92.

Cite this article as: Tran Cao HS, Vauthey JN. Repeat hepatectomy for recurrent colorectal cancer liver metastases after two-stage hepatectomy-limitations and opportunities. Hepatobiliary Surg Nutr 2020;9(1):65-66. doi: 10.21037/ hbsn.2019.06.07 